\title{
Inhibitory effect of burdock leaves on elastase and tyrosinase activity
}

\author{
CHI-TING HORNG ${ }^{1-3^{*}}$, HSING-CHEN WU ${ }^{4 *}$, NI-NA CHIANG ${ }^{5}$, CHIU-FANG LEE ${ }^{5}$, \\ YU-SYUAN HUANG ${ }^{3}$, HUI-YUN WANG ${ }^{3}$, JAI-SING YANG ${ }^{6}$ and FU-AN CHEN ${ }^{3}$
}

\begin{abstract}
${ }^{1}$ Department of Ophthalmology, Kaohsiung Armed Forces General Hospital, Kaohsiung 80284; ${ }^{2}$ Institute of Biochemistry and Biotechnology, Chung Shan Medical University Hospital, Taichung 40201; ${ }^{3}$ Department of Pharmacy and Master Program, Tajen University, Pingtung 90741; ${ }^{4}$ Department of Nutrition, Kaohsiung Armed Forces General Hospital, Kaohsiung 80284;

${ }^{5}$ Department of Pharmacy, Kaohsiung Veterans General Hospital, Pingtung 91245; ${ }^{6}$ Department of Medical Research, China Medical University Hospital, China Medical University, Taichung 40447, Taiwan, R.O.C.
\end{abstract}

Received April 19, 2016; Accepted March 24, 2017

DOI: $10.3892 / \mathrm{etm} .2017 .4880$

\begin{abstract}
Burdock (Arctium lappa L.) leaves generate a considerable amount of waste following burdock root harvest in Taiwan. To increase the use of burdock leaves, the present study investigated the optimal methods for producing burdock leaf extract (BLE) with high antioxidant polyphenolic content, including drying methods and solvent extraction concentration. In addition, the elastase and tyrosinase inhibitory activity of BLE was examined. Burdock leaves were dried by four methods: Shadow drying, oven drying, sun drying and freeze-drying. The extract solution was then subjected to total polyphenol content analysis and the method that produced BLE with the highest amount of total antioxidant components was taken forward for further analysis. The 1,1-diphenyl-2-pycrylhydrazyl scavenging, antielastase and antityrosinase activity of the BLE were measured to enable the evaluation of the antioxidant and skin aging-associated enzyme inhibitory activities of BLE. The results indicated
\end{abstract}

Correspondence to: Dr Fu-An Chen, Department of Pharmacy and Master Program, Tajen University, 20 Wei-Shin Road, Yanpu, Pingtung 90741, Taiwan, R.O.C.

E-mail: fachen.tajen@yahoo.com.tw

Dr Jai-Sing Yang, Department of Medical Research, China Medical University Hospital, China Medical University, 2 Yuh-Der Road, Taichung 40447, Taiwan, R.O.C.

E-mail: jaisingyang@gmail.com

Abbreviations: BLE, burdock leaf extract; EtOH, ethanol; ROS, reactive oxygen species; CHA, chlorogenic acid; DPPH, 1,1-diphenyl-2-pycrylhydrazyl; SANA, N-Suc-(Ala)3-nitroanilide

*Contributed equally

Key words: Arctium lappa, burdock leaves, antioxidant, antityrosinase, antielastase that the total polyphenolic content following extraction with ethanol (EtOH) was highest using the freeze-drying method, followed by the oven drying, shadow drying and sun drying methods. BLE yielded a higher polyphenol content and stronger antioxidant activity as the ratio of the aqueous content of the extraction solvent used increased. BLE possesses marked tyrosinase and elastase inhibitory activities, with its antielastase activity notably stronger compared with its antityrosinase activity. These results indicate that the concentration of the extraction solvent was associated with the antioxidant and skin aging-associated enzyme inhibitory activity of BLE. The reactive oxygen species scavenging theory of skin aging may explain the tyrosinase and elastase inhibitory activity of BLE. In conclusion, the optimal method for obtaining BLE with a high antioxidant polyphenolic content was freeze-drying followed by $30-50 \%$ EtOH extraction. In addition, the antielastase and antityrosinase activities of the BLE produced may be aid in the development of skincare products with antiwrinkle and skin-evening properties.

\section{Introduction}

The skin is the largest organ of the human body and has essential functions, including protecting internal organs and regulating body temperature. Collagen and elastin, which are naturally occurring structural proteins in the dermis of the skin, maintain the plumpness, flexibility and elasticity of the skin. Skin aging presents as wrinkling, roughness, dryness, decreased elasticity and uneven pigmentation $(1,2)$. In addition to intrinsic aging, extrinsic aging caused by exposure of the skin to solar radiation is an important concern. Reactive oxygen species (ROS) caused by ultraviolet (UV) radiation are clinically associated with specific markers of photoaging, including wrinkling, and increased elastin and collagen damage (2,3). Elastase digests elastin (4,5). Pigmentary changes due to the accumulation of melanin are another major characteristic of photoaging. Tyrosinase is a key enzyme that catalyzes melanin biosynthesis in melanocytes $(4,6)$. Previous studies have identified a significant association between the activities of topical treatments, including anti-aging, skin 
evening, and anti-wrinkling, and decreased elastase and tyrosinase activity $(4,5,7)$.

Burdock (Arctium lappa L.), a biennial plant belonging to the Compositae family, has been cultivated in Eastern Asian countries, particularly in Taiwan, China and Japan for use in cooking and in folk medicine as a diuretic, antipyretic and blood purifying agent (8-10). Pharmacologically, burdock has been identified to possess hepatoprotective $(11,12)$, desmutagenic (13), antibacterial $(14,15)$, gastroprotective $(16,17)$, anti-hypoglycemic $(18,19)$, anti-hypolipidemic $(20)$, anti-inflammatory (21), antifatigue (22), antioxidant $(10,21,23)$, body weight management (24) and aphrodisiac (25) activities. Therefore, burdock-containing health drinks and foods are widely consumed in Taiwan. However, burdock leaves are discarded following burdock root harvest, contributing to environmental waste.

Studies investigating the biological activities and application of burdock leaves are warranted. Although burdock leaves have been reported to possess antioxidant and antiproliferative activities (26-28), little is known about their inhibitory effect on skin aging-associated enzymes. Therefore, the present study investigated the potential inhibitory effect of burdock leaves on elastase and tyrosinase activity. In addition, to increase the use of burdock leaves the present study investigated processes for optimizing their polyphenolic content.

\section{Materials and methods}

Materials. Five kilograms Taiwan Good Agriculture Practice (TGAP) certified burdock leaves were obtained from Kang-Li burdock farm in the Gueilai region of Pingtung in Taiwan. Gallic acid, chlorogenic acid (CHA), 1,1-diphenyl-2-pycrylhydrazyl (DPPH), L-3,4-dihydroxyphenylalanine (L-DOPA), $\mathrm{N}$-Suc-(Ala) ${ }_{3}$-nitroanilide (SANA), mushroom tyrosinase, porcine pancreatic elastase and Folin-Ciocalteu reagent were purchased from Sigma-Aldrich (Merck KGaA, Darmstadt, Germany). Liquid chromatography grade methanol and glacial acetic acid were purchased from Macron Fine Chemicals (Avantor Performance Materials, Center Valley, PA, USA). A Cosmosil $5 \mathrm{C}_{18}$-MS-II reverse-phase high performance liquid chromatography (HPLC) column (4.6x250 mm internal diameter; Nakalai Tesque, Inc., Kyoto, Japan) was used for HPLC. Ethanol and phosphoric acid were of analytical reagent grade.

Preparation of the burdock leaf extract (BLE). Fresh burdock leaves were dried through four methods: Sun drying, shadow drying, oven drying and freeze-drying. For sun drying, fresh leaves were exposed directly to sunlight for $16 \mathrm{~h}$. In shadow drying, fresh leaves were dried at an ambient temperature of $27^{\circ} \mathrm{C}$ and a relative humidity of $39 \%$ for $72 \mathrm{~h}$. For oven drying, fresh leaves were dried in an oven at $60^{\circ} \mathrm{C}$ for $16 \mathrm{~h}$. In freeze-drying, a lyophilizer (cat no. 8530023; FD-series, Panchun, Taipei, Taiwan; http://www.panchum.com. tw/product_show.asp?id=1038) was used to dry the burdock leaves for $72 \mathrm{~h}$ at $-80^{\circ} \mathrm{C}$. The four types of BLE $(30 \mathrm{~g}$ of each) were separately added to $600 \mathrm{ml}$ of ethanolic aqueous solution [30, 50 or $95 \%$ ethanol $(\mathrm{EtOH})]$ and refluxed for $3 \mathrm{~h}$ in a reflux extraction apparatus (cat no. 203-330; Angu, Kaohsiung, Taiwan). Subsequently, the extract solution was filtered and the total polyphenol content was prescreened, as described below. The solution from each drying method with the highest amount of total antioxidant components was lyophilized to obtain the BLE at $-80^{\circ} \mathrm{C}$ for $24 \mathrm{~h}$, which was stored in an Eureka Auto Dry Box at $25^{\circ} \mathrm{C}$ and $40 \%$ relative humidity (AD-88S; Taiwan Dry Tech Corp., Taipei, Taiwan) until required for analysis.

Determination of total polyphenol content. The total polyphenol content was estimated spectrophotometrically using Folin-Ciocalteu reagent based on a colorimetric oxidation/reduction reaction, as described previously $(22,29)$ with minor modifications. Briefly, BLE solution $(20 \mu \mathrm{l})$ obtained through the different drying methods, $100 \mu 10.2 \mathrm{~N}$ Folin-Ciocalteu reagent and $80 \mu 17.5 \% \mathrm{Na}_{2} \mathrm{CO}_{3}$ were added to each well of a 96-well microplate and mixed thoroughly. The solution was left to stand for $30 \mathrm{~min}$ at room temperature and the absorbance was measured at $765 \mathrm{~nm}$ using a microplate reader. The total polyphenol content was calculated as the gallic acid equivalent (GAE) on the basis of a calibration curve of gallic acid standard and expressed in $\mathrm{mg} / \mathrm{ml}$, as previously described (22).

DPPH scavenging activity. BLE antioxidant activity was evaluated on the basis of the scavenging capacity toward a stable DPPH free radical as described previously $(22,30,31)$. A stock solution of BLE $(1 \mathrm{mg} / \mathrm{ml})$ was prepared and diluted with methanol to a suitable concentration at $0.5 \mathrm{mg} / \mathrm{ml}$. An aliquot of $50 \mu \mathrm{l}$ of each dilution was transferred into the wells of a 96-well microplate. A working solution of DPPH $(250 \mu \mathrm{M})$ in methanol was freshly prepared and an aliquot of $150 \mu \mathrm{l}$ was added to each well. After incubation for $30 \mathrm{~min}$ at $25^{\circ} \mathrm{C}$, the remaining DPPH was measured at $490 \mathrm{~nm}$ using an ELISA reader. The antioxidant activity was expressed as the DPPH scavenging percentage, as previously described $(22,31)$.

HPLC assay. The expression of CHA (which is an antioxidant component) in BLE was measured using an HPLC assay as described previously (10). Briefly, HPLC analyses were performed using an HPLC system equipped with an L2130 HPLC pump, an L2450 photodiode detector, an L2200 autosampler (Hitachi, Ltd., Tokyo, Japan) and a Cosmosil $5 \mathrm{C}_{18}$-MS-II reverse-phase HPLC column. A mixture of methanol/water/glacial acetic acid (30:69:1, v/v; Sigma-Aldrich; Merck $\mathrm{KGaA}$ ) was used as the mobile phase. A wavelength of $320 \mathrm{~nm}$ was used for monitoring the phenolic compounds and $\mathrm{CHA}$. The flow rate and injection volume were set to $1 \mathrm{ml} / \mathrm{min}$ and $10 \mu \mathrm{l}$, respectively.

Elastase inhibition assay. The effect of BLE on porcine pancreatic elastase activity was estimated spectrophotometrically using SANA as the substrate according to the method described by Moon et al (4) with slight modifications. The release of $p$-nitroaniline was monitored for $15 \mathrm{~min}$ at $25^{\circ} \mathrm{C}$ by measuring the absorbance at $410 \mathrm{~nm}$ on a microplate reader. The reaction was performed in a mixture containing $0.2 \mathrm{M}$ Tris- $\mathrm{HCl}$ buffer ( $\mathrm{pH} 8.0$ ), $10 \mu \mathrm{g} / \mathrm{ml}$ elastase, $5 \mathrm{mM}$ SANA and the test sample $(5 \mathrm{mg} / \mathrm{ml})$ in a 96 -well microplate. Each test sample was preincubated for $10 \mathrm{~min}$ at $25^{\circ} \mathrm{C}$; the reaction commenced upon adding the substrate. The inhibition of elastase was calculated as follows: Inhibition $(\%)=[(\mathrm{A}-\mathrm{B}) / \mathrm{A}] \times 100$, 
where $\mathrm{A}$ and $\mathrm{B}$ were the absorbance at $410 \mathrm{~nm}$ without and with the test sample, respectively.

Tyrosinase inhibition assay. The tyrosinase inhibition activity of BLE was determined by spectrophotometrically measuring the rate of dopachrome formation using L-DOPA as the substrate according to the method described by Choi et al (32) with slight modifications. Briefly, L-DOPA (15 mM; $40 \mu \mathrm{l})$, phosphate buffer ( $\mathrm{pH} 6.8 ; 67 \mathrm{mM} ; 80 \mu \mathrm{l}$ ) and $40 \mu \mathrm{l}$ of either the same buffer or the test sample $(5 \mathrm{mg} / \mathrm{ml})$ were added to each well of a 96-well microplate. Subsequently, $40 \mu 1$ mushroom tyrosinase (125 units) was added to the reaction mixture. After incubation at $37^{\circ} \mathrm{C}$ for $30 \mathrm{~min}$, this reaction mixture was evaluated for the formation of dopachrome using a microplate reader, by measuring the linear increase in the optical density at $492 \mathrm{~nm}$. The tyrosinase inhibition was calculated as follows: Inhibition $(\%)=[(\mathrm{A}-\mathrm{B}) / \mathrm{A}] \mathrm{x} 100$, where $\mathrm{A}$ and $\mathrm{B}$ were the absorbance at $492 \mathrm{~nm}$ without and with the test substance, respectively.

Statistical analysis. The results are presented as the mean \pm standard deviation (SD) of triplicate experiments. The statistical difference between the results of different groups was analyzed using one-way analysis of variance and a post hoc Duncan's new multiple range test. SPSS software (version 10.0; SPSS, Inc., Chicago, IL, USA) was used for all statistical analyses. $\mathrm{P}<0.05$ was considered to indicate a statistically significant difference.

\section{Results}

Polyphenolic content of BLE. Four drying treatments for fresh burdock leaves were assessed; shadow drying, oven drying, sun drying and freeze-drying, and the total polyphenol content of the resulting BLEs was analyzed. As illustrated in Fig. 1, the total polyphenolic content following extraction with $95 \%$ EtOH was highest using the freeze-drying method, followed by the oven drying, shadow drying and sun drying methods. The polyphenolic content obtained from the freeze-drying method was significantly ( $\sim 3$ times) increased compared with that obtained through the other methods tested $(\mathrm{P}<0.05$; Fig. 1$)$. The effect of the concentration of ethanolic solvent used for extraction on the polyphenol content of BLE was investigated in the present study. As depicted in Fig. 2, freeze-drying the BLEs following extraction using $95 \% \mathrm{EtOH}$ yielded a significantly lower polyphenolic content (GAE) compared with solvents extracted using 30 and $50 \%$ EtOH $(\mathrm{P}<0.05)$, indicating that an increase in the aqueous ratio results in a higher polyphenol content.

BLE has DPPH free radical scavenging activity. A DPPH-scavenging assay was used to measure the antioxidant capacity of the BLE obtained using different concentrations of EtOH as presented in Fig. 3. A similar effect was observed, with BLE extracted using 30 and $50 \% \mathrm{EtOH}$ yielding a higher DPPH savaging ability compared with BLE extracted using $95 \% \mathrm{EtOH}$.

Antityrosinase and antielastase activities of the BLE. CHA is an antioxidant component of burdock leaves $(26,27)$. As

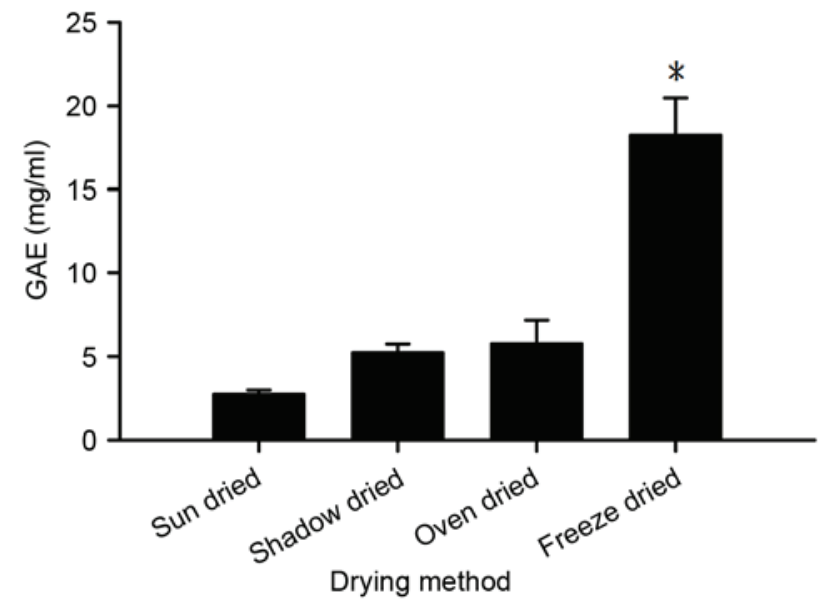

Figure 1. Polyphenolic content of BLE produced from different drying methods. GAE, gallic acid equivalent. "P<0.05 vs. sun dried, shadow dried and oven-dried samples. The results are presented as the mean \pm standard deviation of triplicate experiments.

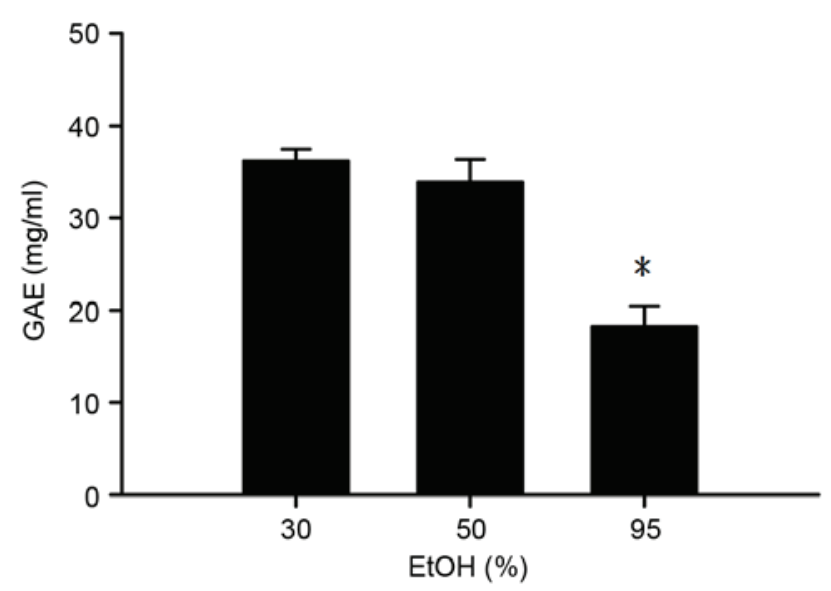

Figure 2. Polyphenolic content of the BLE obtained using different concentrations of $\mathrm{EtOH}$ for extraction following freeze-drying. ${ }^{*} \mathrm{P}<0.05$ vs. 30 and $50 \% \mathrm{EtOH}$. The results are presented as the mean \pm standard deviation of triplicate experiments. GAE, gallic acid equivalent; EtOH, ethanol.

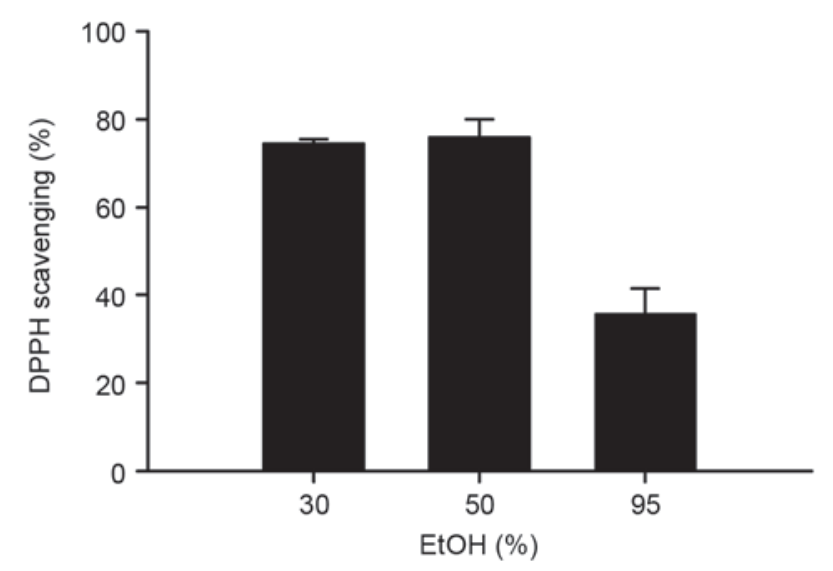

Figure 3. DPPH free radical scavenging activity of the BLE obtained using different concentrations of EtOH for extraction following freeze-drying. The free radical scavenging activity was evaluated as the DPPH scavenging percentage in the presence of BLE for $30 \mathrm{~min}$. The results are presented as the mean \pm standard deviation of triplicate experiments. BLE, burdock leaf extract; EtOH, ethanol; DPPH, 1,1-diphenyl-2-pycrylhydrazyl. 


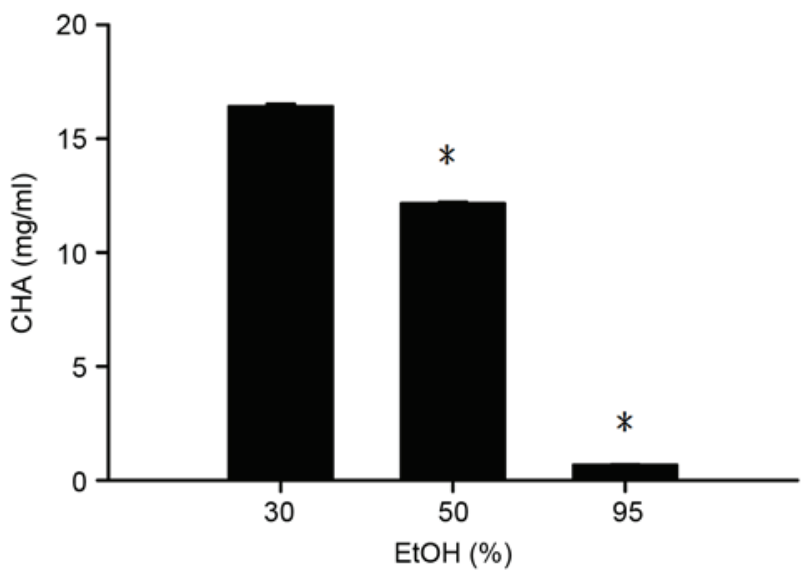

Figure 4. CHA content of the BLE obtained using different concentrations of EtOH for extraction following freeze-drying. "P $<0.05$ vs. $30 \% \mathrm{EtOH}$. The results are presented as the mean \pm standard deviation of triplicate experiments. CHA, chlorogenic acid; EtOH, ethanol.

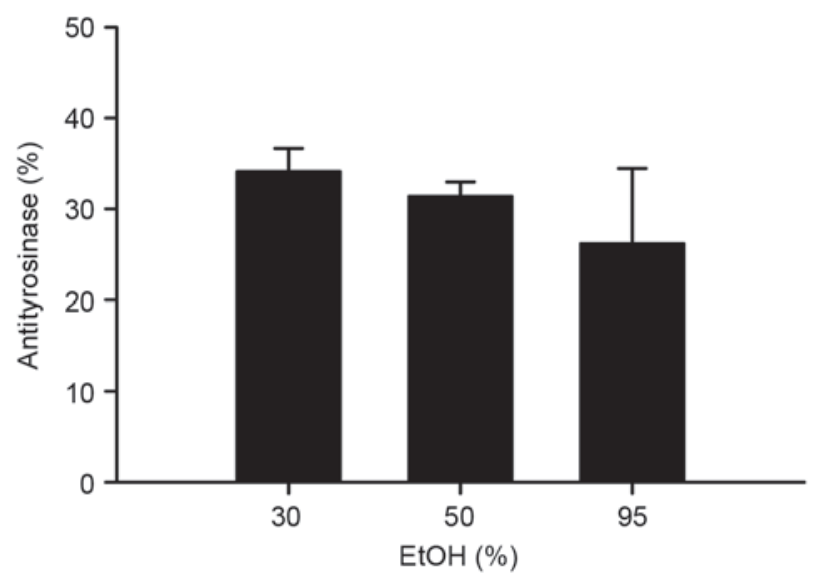

Figure 5. Antityrosinase activity of the BLE obtained using different concentrations of EtOH for extraction following freeze-drying. The results are presented as the mean \pm standard deviation of triplicate experiments. EtOH, ethanol.

observed in Fig. 4, freeze-drying BLE following extraction using 50 and $95 \%$ EtOH resulted in a significantly lower $\mathrm{CHA}$ content compared with solvents extracted using $30 \% \mathrm{EtOH}$ $(\mathrm{P}<0.05)$. This indicates that an increase in the ratio of ethanol negatively affects $\mathrm{CHA}$ extraction. The trend observed in the CHA content in the BLE using different concentrations of $\mathrm{EtOH}$ for extraction was only marginally similar to those for polyphenolic content and antioxidant activity. This suggests that polyphenolic components other than $\mathrm{CHA}$ contribute to polyphenolic content and antioxidant activity.

As depicted in Fig. 5, the BLEs produced following the use of 30,50 and $95 \% \mathrm{EtOH}$ exhibited a marked tyrosinase inhibitory effect ( $>25 \%$ inhibition). There was no significant difference in tyrosinase inhibition between the groups. In addition, the BLEs obtained using 30 and 50\% EtOH notably inhibited elastase activity $(>60 \%)$, and this inhibition was significantly increased, compared with that if the BLE obtained using 95\% EtOH ( $\mathrm{P}<0.05$; Fig. 6). These results indicate that the antielastase activity of BLE is higher compared with its antityrosinase activity.

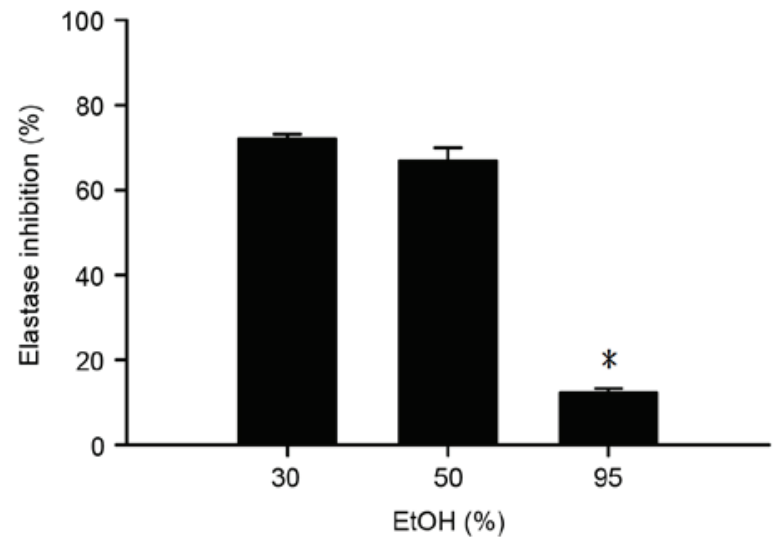

Figure 6. Antielastase activity of the BLE obtained using different concentrations of $\mathrm{EtOH}$ for extraction following freeze-drying. ${ }^{*} \mathrm{P}<0.05$ vs. 30 and $50 \% \mathrm{EtOH}$. The results are presented as the mean \pm standard deviation of triplicate experiments. EtOH, ethanol.

\section{Discussion}

Burdock is cultivated for dietary and traditional medicinal use in Eastern Asian countries (8-10). In Taiwan, burdock leaves are discarded following burdock root harvest, contributing to environmental waste. To increase the number of applications for these leaves, the present study investigated processes for optimizing their polyphenolic content, including certain drying and solvent extraction methods. In addition, the inhibitory activity of skin aging-associated enzymes in burdock leaves was examined in the current study.

Four drying treatments for fresh burdock leaves were determined. The polyphenolic content obtained from the freeze-drying method was significantly increased compared to the other methods assessed. These findings are consistent with previous reports, which identified that temperature strongly influences polyphenolic stability $(10,33)$. Furthermore, these results indicate that freeze-drying is the optimal method of maintaining the polyphenolic content of burdock leaves; therefore, this method was applied to obtain the extracts used in subsequent experiments. The polyphenolic components other than CHA contribute to polyphenolic content and antioxidant activity. This is consistent trend with previous results $(33,34)$, which reported that ethanol and methanol with $50-70 \%$ water content improved the extraction of antioxidant compounds from plants.

Skincare products formed the largest proportion $(\sim 23 \%)$ of cosmetics sales from 1998-2010 worldwide (35). Increased wrinkling, decreased elasticity, uneven pigmentation, and increased roughness and dryness are common signs of skin aging $(1,2)$. Plants with natural antioxidant components that possess antiaging activities may have cosmetic applications. Polyphenols with antioxidant activity are frequently present in plants and are associated with various biological functions, including inhibiting skin aging-associated enzymes (36-38). Burdock leaves have recently been reported to contain polyphenols with antioxidant activity $(10,26,27)$. For burdock leaves to be used effectively the following procedures are required: Measuring their content of antioxidant polyphenols; determining their inhibitory activity on skin aging-associated enzymes; and optimizing BLE preparation methods, including 
drying and solvent extraction. The results of the current study indicate that the extraction of burdock leaves with $30-50 \%$ $\mathrm{EtOH}$ following freeze-drying is the optimal method for extracting antioxidant polyphenols.

ROS formed due to various extrinsic environmental factors and intrinsic endogenous metabolism are the most common causes of skin aging. UV radiation activates elastase secretion via ROS formation, resulting in a decreased elastin content, leading to wrinkles $(2,3,33)$. The inhibition of skin elastase and tyrosinase serves a key role in the antiwrinkle and skin-evening activities (4,7,33). Antioxidant polyphenols possess tyrosinase and elastase inhibitory activity $(4,36,39)$. In the current study, the extract from burdock leaves with antioxidant polyphenols inhibited elastase and tyrosinase activity, with their antielastase activity being stronger compared with their antityrosinase activity, which can be explained using the ROS scavenging theory of skin aging (40). These results indicate that the antiwrinkle activity of burdock leaves may be more effective than its skin-evening activity.

In conclusion, the current study demonstrated that BLE contains polyphenols, including CHA, and exhibits antioxidant activity. In addition, the BLE produced possessed marked tyrosinase and elastase inhibitory activity, which were associated with its polyphenolic content and antioxidant activities. Freeze drying followed by $30-50 \% \mathrm{EtOH}$ extraction was identified to be the optimal method for obtaining BLE with high levels of antioxidant polyphenols. The antielastase and antityrosinase activity of BLE may be beneficial for developing skincare products with antiwrinkle and skin-evening activities. Further research is required to clarify the individual antioxidant components and the underlying pharmacological mechanisms of these effects of BLE.

\section{Acknowledgements}

The present study was supported by Kaohsiung Armed Forces General Hospital (Kaohsiung, Taiwan).

\section{References}

1. Gilchrest BA: Skin aging and photoaging: An overview. J Am Acad Dermatol 21: 610-613, 1989.

2. Waqas MK, Akhtar N, Rasul A, Rashid SU, Mustafa R, Khan BA and Murtaza G: In vivo evaluation of a cosmetic emulsion containing soybean extract for anti-aging. Trop J Pharm Res 13 1401-1406, 2014

3. Ryu J, Park SJ, Kim IH, Choi YH and Nam TJ: Protective effect of porphyra-334 on UVA-induced photoaging in human skin fibroblasts. Int J Mol Med 34: 796-803, 2014.

4. Moon JY, Yim EY, Lee NO and Hyun CG: Screening of elastase and tyrosinase inhibitory activity from Jeju Island plants. Eur Asia J BioSci 4: 41-53, 2010.

5. Azmi N, Hashim P, Hashim DM, Halimoon N and Majid NM: Anti-elastase, anti-tyrosinase and matrix metalloproteinase-1 inhibitory activity of earthworm extracts as potential new anti-aging agent. Asian Pac J Trop Biomed 4 (Suppl 1): S348-S352, 2014.

6. Chang TS: Natural melanogenesis inhibitors acting through the down-regulation of tyrosinase activity. Materials 5: 1661-1685, 2012.

7. Jamaluddin A, Rashid NY, Razak DL, Sharifudin SA and Long K: Effect of fungal fermentation on tyrosinase and elastase inhibition activity in rice bran. Agr Agr Sci Proced 2: 252-256, 2014.

8. Kan WS: Pharmaceutical Botany. National Research Institute of Chinese Medicine, 1993.
9. Han CM: Cultivation management of burdock in Kao-Ping Area. Agriculture World 145: 55-57, 1995.

10. Chen FA, Wu AB and Chen CY: The influence of different treatments on the free radical scavenging activity of burdock and variations of its active components. Food Chem 86: 479-484, 2004.

11. Lin SC, Chung TC, Lin CC, Ueng TH, Lin YH, Lin SY and Wang LY: Hepatoprotective effects of Arctium lappa on carbon tetrachloride- and acetaminophen-induced liver damage. Am J Chinese Med 28: 163-173, 2000.

12. Lin SC, Lin CH, Lin CC, Lin YH, Chen CF, Chen IC and Wang LY: Hepatoprotective effects of Arctium lappa Linne on liver injuries induced by chronic ethanol consumption and potentiated by carbon tetrachloride. J Biomed Sci 9: 401-409, 2002.

13. Morita K, Kada T and Namiki M: A desmutagenic factor isolated from burdock (Arctium lappa Linne). Mutat Res 129: 25-31, 1984.

14. Chow LW, Wang SJ and Duh PD: Antibacterial activity of burdock. Food Sci 24: 195-202, 1997.

15. Holetz FB, Pessini GL, Sanches NR, Cortez DA, Nakamura CV and Dias BP: Screening of some plants used in the Brazilian folk medicine for the treatment of infectious diseases. Mem Inst Oswaldo Cruz 97: 1027-1031, 2002.

16. Dos Santos AC, Baggio CH, Freitas CS, Lepieszynski J, Mayer B, Twardowschy A, Missau FC, dos Santos EP, Pizzolatti MG and Marques MC: Gastroprotective activity of the chloroform extract of the roots from Arctium lappa L. J Pharm Pharmacol 60: 795-801, 2008.

17. Chen FA, Lee SC, Chao HR, Fu WC, Hsu MC, Horng CT, Wang CC, Matsui $\mathrm{H}$ and Agoramoorthy G: Effects of burdock extract preparation on gastric mucosal protection. Asian J Chem 21: 3015-3022, 2009.

18. Cao JF, Li CP, Zhang PY, Cao X, Huang T, Bai Y and Chen K: Antidiabetic effect of burdock (Arctium lappa L.) root ethanolic extract on streptozotocin-induced diabetic rats. African J Biotechnol 11: 9079-9085, 2012.

19. Chang TH, Liu IM, Horng CT, Tsai FC, Kuo DH, Shieh PC, Lee SC, Shiang JC and Chen FA: Beneficial effects of the burdock ferment liquid on diabetic disorders in STZ-induced diabetic rats. Life Sci J 9: 823-831, 2012.

20. Lee YJ, Choi DH, Cho GH, Kim JS, Kang DG and Lee HS: Arctium lappa ameliorates endothelial dysfunction in rats fed with high fat/cholesterol diets. BMC Complement Altern Med 12: 116, 2012

21. Lin CC,Lu JM, Yang JJ, Chuang SC and UjiieT: Anti-inflammatory and radical scavenge effects of Arctium lappa. Am J Chin Med 24: 127-137, 1996.

22. Horng CT, Tsai ML, Hsueh CW, Hsu SY, Wang HY and Chen FA: Antioxidant activity of Arctium lappa L. and its effect on biochemical parameters in exercised rats. Asian J Chem 25: 1970-1974, 2013

23. Duh PD: Antioxidant activity of burdock (Arctium lappa Linne): Its scavenging effect on free-radical and active oxygen. J Am Oil Chem Soc 75: 455-461, 1998.

24. Kuo DH, Hung MC, Hung CM, Liu LM, Chen FA, Shieh PC, Ho CT and Way TD: Body weight management effect of burdock (Arctium lappa L.) root is associated with the activation of AMP-activated protein kinase in human HepG2 cells. Food Chem 134: 1320-1326, 2012.

25. JianFeng C, PengYing Z, ChengWei X, TaoTao H, YunGui B and KaoShan C: Effect of aqueous extract of Arctium lappa $\mathrm{L}$. (burdock) roots on the sexual behavior of male rats. BMC Complement Altern Med 12: 8, 2012.

26. Lou Z, Wang H, Li J, Chen S, Zhu S, Ma C and Wang Z: Antioxidant activity and chemical composition of the fractions from burdock leaves. J Food Sci 75: C413-C419, 2010.

27. Lou Z, Wang H, Zhu S, Zhang M, Gao Y, Ma C and Wang Z: Improved extraction and identification by ultra performance liquid chromatography tandem mass spectrometry of phenolic compounds in burdock leaves. J Chromatogr A 1217: 2441-2446, 2010.

28. Machado FB, Yamamoto RE, Zanoli K, Nocchi SR, Novello CR, Schuquel IT, Sakuragui CM, Luftmann H, Ueda-Nakamura T, Nakamura CV and de Mello JC: Evaluation of the antiproliferative activity of the leaves from Arctium lappa by a bioassay-guided fractionation. Molecules 17: 1852-1859, 2012.

29. Negi PS, Jayaprakasha GK and Jena BS: Antioxidant and antimutagenic activities of pomegranate peel extracts. Food Chem 80: 393-397, 2003.

30. Blois MS: Antioxidant determinations by the use of a stable free radical. Nature 181: 1199-1200, 1958. 
31. Chen FA, Wu AB, Shieh P, Kuo DH and Hsieh CY: Evaluation of the antioxidant activity of Ruellia tuberosa. Food Chem 94: 14-18, 2006.

32. Choi SY, Kim S, Kim H, Suk K, Hwang JS, Lee BG, Kim AJ and Kim SY: (4-methoxy-benzylidene)-(3-methoxy-phenyl)-amine, a nitrogen analog of stilbene as a potent inhibitor of melanin production. Chem Pharm Bull (Tokyo) 50: 450-452, 2002.

33. Anwar F, Kalsoom U, Sultana B, Mushtaq M, Mehmood T and Arshad HA: Effect of drying method and extraction solvent on the total phenolics and antioxidant activity of cauliflower (Brassica oleracea L.) extracts. Int Food Res J 20: 653-659, 2013.

34. Sultana B, Anwar F and Ashraf M: Effect of extraction solvent/technique on the antioxidant activity of selected medicinal plant extracts. Molecules 14: 2167-2180, 2009.

35. Lopaciuk A and Loboda M: Global beauty industry trends in the 21st century. Management Knowledge Learning: 1079-1087, 2013.

36. Karim AA, Azlan A, Ismail A, Hashim P, Abd Gani SS, Zainudin BH and Abdullah NA: Phenolic composition, antioxidant, anti-wrinkles and tyrosinase inhibitory activities of cocoa pod extract. BMC Complement Altern Med 14: 381, 2014.
37. Wittenauer J, Mackle S, Sussmann D, Schweiggert-Weisz U and Carle R: Inhibitory effects of polyphenols from grape pomace extract on collagenase and elastase activity. Fitoterapia 101: 179-187, 2015.

38. Du ZY, Jiang YF, Tang ZK, Mo RQ, Xue GH, Lu YJ, Zheng X, Dong $C Z$ and Zhang K: Antioxidation and tyrosinase inhibition of polyphenolic curcumin analogs. Biosci Biotechnol Biochem 75: 2351-2358, 2011

39. Onar HC, Yusufoglu A, Turker G and Yanardag R: Elastase, tyrosinase and lipoxygenase inhibition and antioxidant activity of an aqueous extract from Epilobium angustifolium L. leaves. J Med Plant Res 6: 716-726, 2012.

40. Rinnerthaler M, Bischof J, Streubel MK, Trost A and Richter K: Oxidative stress in aging human skin. Biomolecules 5: 545-589, 2015. 